\title{
The Split-Weight Particle Simulation Scheme for Plasmas
}

\author{
Igor Manuilskiy and W. W. Lee \\ Plasma Physics Laboratory, Princeton University, Princeton, NJ 08543
}

\begin{abstract}
An efficient numerical method for treating electrons in magnetized plasmas has been developed. The scheme, which is based on the perturbative $(\delta f)$ gyrokinetic particle simulation, splits the particle electron responses into adiabatic and non-adiabatic parts. The former is incorporated into the gyrokinetic Poisson's equation, while the latter is calculated dynamically with the aid of the charge conservation equation. The new scheme affords us the possibility of suppressing unwanted high frequency oscillations and, in the meantime, relaxing the Courant condition for the thermal particles moving in the parallel direction. It is most useful for studying low-frequency phenomena in plasmas. As an example, one-dimensional drift wave simulation has been carried out using the scheme and the results are presented in the paper. This methodology can easily be generalized to problems in three-dimensional toroidal geometry as well as those in unmagnetized plasmas.
\end{abstract}


It has been shown by Langdon [1] that, in particle simulation, a thermal particle traversing a distance longer than $L / 2 \pi$ in one time step will result in incorrect plasma response, where $L$ is the wavelength of interest. However, in simulating low-frequency microinstabilities, one often faces the unique property of $k_{\|} v_{t i} \ll \omega \ll k_{\|} v_{t e}$, where $\omega$ is the frequency of interest and $v_{t \alpha}$ is the thermal velocity of the species $\alpha$. Thus, the time step requirement of $k_{\|} v_{t e} \Delta t<1$ imposed by the electrons is unnecessarily stringent than the stability condition of $\omega \Delta t<1$. However, we know that fast electrons respond adiabatically to the perturbations and only the slow electrons interact with the waves. The scheme described in this paper is an attempt to capture this unique feature and to increase the time steps, if desirable.

To accomplish this, we resort to the recently developed perturbative $(\delta f)$ particle simulation methods $[2,3]$. The reason is that, by following the perturbed part of the distribution $\delta f$ ( $=F-F_{0}$, where $F$ and $F_{0}$ are the total and equilibrium distributions, respectively), we are able to determine beforehand which individual particles will respond adiabatically to the waves. Dimits and Lee [2] first developed the cut-off scheme in slab geometry for that purpose by specifying a cut-off velocity in the parallel direction, above which $\delta f / F_{0} \approx e \phi / T_{e}$. As we will show, the split-weight scheme is an improvement and is easily generalized to general geometry cases.

Let us first start with the gyrokinetic Vlasov-Poisson system [4]. The gyrokinetic Vlasov equation in slab geometry for $k_{\perp}^{2} \rho_{i}^{2} \ll 1$ takes the form of

$$
\frac{d F_{\alpha}}{d t} \equiv \frac{\partial F_{\alpha}}{\partial t}+v_{\|} \frac{\partial F_{\alpha}}{\partial x_{\|}}-\frac{c}{B} \frac{\partial \phi}{\partial \mathbf{x}} \times \hat{\mathbf{b}} \cdot \frac{\partial F_{\alpha}}{\partial \mathbf{x}}+s_{\alpha} v_{t \alpha}^{2}\left(\frac{\partial}{\partial x_{\|}} \frac{e \phi}{T_{e}}\right) \frac{\partial F_{\alpha}}{\partial v_{\|}}=0,
$$

where $v_{t \alpha} \equiv \sqrt{T_{\alpha} / m_{\alpha}}, \alpha$ denotes species and $\left(s_{e}, s_{i}\right)=\left(1,-\tau \equiv-T_{e} / T_{i}\right)$ for the electrons and ions, respectively. Letting

$$
F_{\alpha}=F_{0 \alpha}+\delta f_{\alpha}
$$

gives rise to

$$
\frac{d \delta f_{\alpha}}{d t}=-\frac{d F_{0 \alpha}}{d t}=-\frac{c}{B} \frac{\partial \phi}{\partial \mathbf{x}} \times \hat{\mathbf{b}} \cdot \boldsymbol{\kappa}_{\alpha} F_{0 \alpha}+s_{\alpha} v_{\|}\left(\frac{\partial}{\partial x_{\|}} \frac{e \phi}{T_{e}}\right) F_{0 \alpha},
$$


where $\boldsymbol{\kappa}_{\alpha} \equiv-\left(\partial F_{0 \alpha} / \partial \mathbf{x}\right) / F_{0 \alpha}$ represents the zeroth-order inhomogeneity. The corresponding equations of motion for particle pushing are

$$
\begin{gathered}
\frac{d \mathbf{x}}{d t}=v_{\|} \hat{\mathbf{b}}-\frac{c}{B} \frac{\partial \phi}{\partial \mathbf{x}} \times \hat{\mathbf{b}}, \\
\frac{d v_{\|}}{d t}=s_{\alpha} v_{t \alpha}^{2} \frac{\partial}{\partial x_{\|}} \frac{e \phi}{T_{e}}
\end{gathered}
$$

and the weight equation is

$$
\frac{d w}{d t}=\frac{1}{F_{\alpha}} \frac{d \delta f_{\alpha}}{d t}=(1-w)\left(-\frac{c}{B} \frac{\partial \phi}{\partial \mathbf{x}} \times \hat{\mathbf{b}} \cdot \boldsymbol{\kappa}_{\alpha}+s_{\alpha} v_{\|} \frac{\partial}{\partial x_{\|}} \frac{e \phi}{T_{e}}\right),
$$

with the definition of

$$
w=\frac{\delta f_{\alpha}}{F_{\alpha}}
$$

The gyrokinetic Poisson's equation [4] in the limit of $k_{\perp}^{2} \rho_{i}^{2} \ll 1$ can be simplified as

$$
\rho_{s}^{2} \nabla_{\perp}^{2} \frac{e \phi}{T_{e}}=\delta n_{e}-\delta n_{i}
$$

where $\rho_{s}^{2}=\tau \rho_{i}^{2}$,

$$
\delta n_{\alpha}=\int \delta f_{\alpha} d v_{\|}
$$

and $\int F_{0 \alpha} d v_{\|}=1$. This is the standard $\delta f$ particle simulation scheme [3], for which

$$
\delta f=\sum_{j=1}^{N} w_{j} \delta\left(\mathbf{x}-\mathbf{x}_{j}\right) \delta\left(v_{\|}-v_{\| j}\right)
$$

in Klimontovich-Dupree representation, where $N$ is the total number of particles in the system.

The corresponding linear dispersion relation for such a system with cold ions using the ansatz of $\exp \left(i k_{\|} x_{\|}-i \omega t\right)$ can be expressed as

$$
\epsilon \equiv 1+k_{\perp}^{2} \rho_{s}^{2}+X_{e}-\frac{k_{\|}^{2} c_{s}^{2}}{\tau \omega^{2}}=0
$$

where $X_{e} \equiv \xi_{e} Z\left(\xi_{e}\right), Z$ is the usual plasma function, $\xi_{e}=\omega / \sqrt{2} k_{\|} v_{t e}$ and $c_{s}$ is the ion acoustic speed. The electron density response is 


$$
\delta n_{e}=\left(1+X_{e}\right) \frac{e \phi}{T_{e}} .
$$

For the cold plasma response of $\omega \gg k_{\|} v_{t e}, X_{e} \approx-1-\left(k_{\|} v_{t e} / \omega\right)^{2}$ gives the linearly damped high frequency modes as

$$
\omega= \pm \omega_{H} \equiv \pm \frac{k_{\|}}{k_{\perp}} \sqrt{\frac{m_{i}}{m_{e}}} \Omega_{i}
$$

These are the electrostatic version of the shear-Alfven modes [5] and they are responsible for damping the numerical noise generated by the fast-moving electrons with $\delta n_{e} \ll e \phi / T_{e}$. However, they also set the restrictions on the size of the time step in the simulation. For the warm response of $\omega \ll k_{\|} v_{t e}, X_{e} \approx 0$ and $\delta n_{e} \approx e \phi / T_{e}$ yield the ion acoustic branch with

$$
\omega= \pm \omega_{s}= \pm \frac{k_{\|} c_{s}}{\sqrt{\tau\left(1+k_{\perp}^{2} \rho_{s}^{2}\right)}}
$$

They are the waves of interest, specifically, the branch associated with microinstabilities in magnetized plasmas with $k_{\|} \ll k_{\perp}$. The purpose of split-weight scheme is to suppress the high frequency modes and the associated numerical restrictions, while preserving the physics of the long wave-length low-frequency branch.

To proceed, let us first write down the linearized equations of motion for the electrons in a homogeneous plasma. From Eqs. (2)-(4), they become

$$
\begin{aligned}
& \frac{d x_{\|}}{d t}=v_{\|}, \\
& \frac{d v_{\|}}{d t}=0,
\end{aligned}
$$

and

$$
\frac{d w}{d t}=v_{\|} \frac{\partial}{\partial x_{\|}} \frac{e \phi}{T_{e}}
$$

Following the derivation by Langdon [1], we can show that $X_{e}$ in Eq. (6) becomes

$$
X_{e}=-1+\left(k_{\|} v_{t e} \Delta t\right)^{2} \sum_{q=0}^{\infty} q \exp \left[i q \omega \Delta t-q^{2}\left(k_{\|} v_{t e} \Delta t\right)^{2} / 2\right]
$$


when the leap-frog scheme is assumed to push the electrons using the linearized equations of motion, where $\Delta t$ is the time step. It has been shown $[1,5]$ that substituting this expression of $X_{e}$ into the dispersion relation, Eq. (6), with $\omega \Delta t>1$ and $k_{\|} v_{t e} \Delta t<1$ results in numerical instability for the high frequency branch characterized by $\omega \gg k_{\|} v_{t e}$, whereas

$$
k_{\|} v_{t e} \Delta t>1
$$

alone causes only inaccuracy. For example, $X_{e} \approx-1$ for $k_{\|} v_{t e} \Delta t \gg 1$ gives $\delta n_{e} \approx 0$ and the modes of $\omega= \pm\left(k_{\|} / k_{\perp}\right) \Omega_{i} / \sqrt{\tau} \sim \omega_{s} \ll \omega_{H}$. (Note that $k_{\|} \rightarrow 0$ also gives $X_{e} \approx-1$ and $\delta n_{e} \approx 0$.) If we are only interested in low frequency modes with $\omega \ll k_{\|} v_{t e}$, we can conceivably violate the parallel Courant condition by allowing Eq. (12) for large $k_{\|}$modes, while preserving the condition of $w \Delta t<1$ and $k_{\|} v_{t e} \Delta t<1$ for the waves of interest.

However, the $\delta f$ scheme affords us a more versatile approach. Since $d / d t \equiv \partial / \partial t+$ $v_{\|} \partial / \partial x_{\|}$, Eq. (11) gives

$$
w \approx \frac{e \phi(\mathbf{x})}{T_{e}}
$$

for $\omega \ll k_{\|} v_{\|}$, which, in turn, gives $\delta n_{e} \approx e \phi / T_{e}$. Thus, by using this procedure, we can recover the low-frequency branch of $\omega_{s}$ as well as the adiabatic electron response widely used in the community for simulating ion temperature gradient drift instabilities [6,7]. Most of all, we can now tag the response for each individual particle with the $\delta f$ scheme. To capture this salient feature for simulating drift waves, Dimits and Lee [2] developed the cutoff scheme for slab geometry by dividing the electrons into adiabatic particles and non-adiabatic particles according to their parallel velocity. The scheme calculates particle variables using Eqs. (2)(4) or Eqs. (9)-(11) for particles with $v_{\|}<v_{\text {cut }}$, while using only Eq. (9) [or Eq. (2)] and Eq. (10) to follow the particles with $v_{\|}>v_{c u t}$, where $v_{c u t}\left(\ll v_{t e}\right)$ is the cut-off velocity. For this class of fast particles, their responses are automatic given by Eq. (13), where x, e.g., can be the current particle position. The reason for doing so is that calculating the actual fast particle response using Eqs. (3) and (4) or (11) is inaccurate because of $k_{\|} v_{\|} \Delta t>1$. The time step for the slow particles, therefore, is now determined by $k_{\|} v_{c u t} \Delta t<1$ achieving the condition of Eq. (12) for the faster thermal particles. 
The split-weight scheme represents a more general approach than the cut-off scheme. Following the standard procedure in microinstability analyses, we first split the perturbed electron distribution into two parts,

$$
\delta f_{e}=\frac{e \phi}{T_{e}} F_{0 e}+\delta h_{e}
$$

i.e., the adiabatic and non-adiabatic parts, regardless of the parallel velocity. From

$$
\frac{d}{d t} \frac{e \phi}{T_{e}}=\frac{\partial}{\partial t} \frac{e \phi}{T_{e}}+v_{\|} \frac{\partial}{\partial x_{\|}} \frac{e \phi}{T_{e}}
$$

and Eq. (1), we obtain

$$
\frac{d \delta h_{e}}{d t}=-\left(\frac{\partial}{\partial t} \frac{e \phi}{T_{e}}\right) F_{0 e}-\left(1+\frac{e \phi}{T_{e}}\right) \frac{c}{B} \frac{\partial \phi}{\partial \mathbf{x}} \times \hat{\mathbf{b}} \cdot \boldsymbol{\kappa}_{e} F_{0 e}+\frac{v_{\|}}{2}\left[\frac{\partial}{\partial x_{\|}}\left(\frac{e \phi}{T_{e}}\right)^{2}\right] F_{0 e} .
$$

Defining

$$
w^{N A} \equiv \frac{\delta h_{e}}{F_{e}}
$$

the weight equation can then be written as

$$
\frac{d w^{N A}}{d t}=\frac{1}{F_{e}} \frac{d \delta h_{e}}{d t}=\frac{1-w^{N A}}{1+e \phi / T_{e}}\left[-\frac{\partial}{\partial t} \frac{e \phi}{T_{e}}-\left(1+\frac{e \phi}{T_{e}}\right) \frac{c}{B} \frac{\partial \phi}{\partial \mathbf{x}} \times \hat{\mathbf{b}} \cdot \boldsymbol{\kappa}_{e}+\frac{v_{\|}}{2} \frac{\partial}{\partial x_{\|}}\left(\frac{e \phi}{T_{e}}\right)^{2}\right]
$$

The gyrokinetic Poisson's equation is then modified as

$$
\left(\rho_{s}^{2} \nabla_{\perp}^{2}-1\right) \frac{e \phi}{T_{e}}=\delta n_{e}^{N A}-\delta n_{i}
$$

where

$$
\begin{gathered}
\delta n_{e}^{N A}=\int \delta h_{e} d v_{\|}, \\
\delta h_{e}=\sum_{j=1}^{N} w_{j}^{N A} \delta\left(\mathbf{x}-\mathbf{x}_{j}\right) \delta\left(v_{\|}-v_{\| j}\right) .
\end{gathered}
$$

However, serious numerical instabilities may arise from Eq. (15) because of the existence of time derivatives on both sides of the equation. Although for a different reason, this situation is similar to the problem of Darwin model for nonradiative simulation facing Nielson and 
Lewis [8]. To avoid the numerical difficulty, we first take the partial time derivative of Eq. (5) and substitute the $\partial \delta n_{e} / \partial t$ term by using the continuity equation from Eq. (1) to obtain

$$
\rho_{s}^{2} \nabla_{\perp}^{2}\left(\frac{\partial}{\partial t} \frac{e \phi}{T_{e}}\right)=-\frac{\partial \delta u_{e}}{\partial x_{\|}}+\frac{\partial \delta u_{i}}{\partial x_{\|}}+\frac{c}{B} \frac{\partial \phi}{\partial \mathbf{x}} \times \hat{\mathbf{b}} \cdot \frac{\partial}{\partial \mathbf{x}}\left(\rho_{s}^{2} \nabla_{\perp}^{2} \frac{e \phi}{T_{e}}\right),
$$

where

$$
\delta u_{\alpha}=\int v_{\|} \delta f_{\alpha} d v_{\|}
$$

is the parallel fluid velocity for the species $\alpha$. [Note that $\delta u_{e}=\int v_{\|} \delta h_{e} d v_{\|}$.] This equation can be viewed as the charge conservation equation associated with the polarization density. Combining Eqs. (16) and (17) for solving the fields along with Eqs. (2) and (3) for the particle pushing as well as Eq. (4) and Eq. (15) for calculating the particles weights for the ions and electrons, respectively, we have a complete system of equations for the split-weight scheme.

However, Eqs. (3) and (15) contain terms commonly associated with velocity-space nonlinearities and still carry the restriction imposed by $k_{\|} v_{\|} \Delta t \leq 1$ for the electrons. [Eq. (3) can be re-written as $(1 / 2) d\left(v_{\|} / v_{t e}\right)^{2} / d t=v_{\|} \partial\left(e \phi / T_{e}\right) / \partial x_{\|}$.] On the other hand, they are usually negligible in multi-dimensional microturbulence problems [see, for example, Ref. [9]]. Specifically, the two dimensional nonlinear drift waves simulation in slab geometry [10] has shown that the dominant nonlinear mechanism is the $\mathbf{E} \times \mathbf{B}$ advection and can be easily reproduced by using Eqs. (2) and (10) as well as Eq. (15) without the nonlinear term in $v_{\|}$. With these $v_{\|}$terms gone, we can indeed use large time steps specified by Eq. (12) and circumvent the parallel Courant conditions for the fast (thermal) particles. For example, by comparing the original weight equation, Eqs. (4) or (11), with the modified weight equation, Eq. (15), we obtain

$$
\frac{-\partial \phi / \partial t}{v_{\|} \partial \phi / \partial x_{\|}} \simeq \frac{\omega}{k_{\|} v_{\|}}
$$

For typical low frequency modes with $\omega \ll k_{\|} v_{\|}$in comparison with most of the electron velocity distribution, the split weight scheme relaxes the condition of $k_{\|} v_{\|} \Delta t<1$ to a more 
reasonable requirement of $\omega \Delta t<1$. In other words, for the warm electron response of $\omega / k_{\|} v_{t e} \ll 1$, we can indeed violate the Courant condition for the fast particles, Eq. (12). On the other hand, if these nonlinear terms become important, as the case for the one dimensional drift waves [3], the Courant conditions for the modes of interest will have to be satisfied. But, if we are only interested in the linear one dimensional simulation, the Courant condition can again be violated.

Another interesting feature of the split weight scheme can be ascertained by using the same procedure in obtaining Eq. (13) to Eq. (15) and obtain

$$
w^{N A} \approx \frac{\omega}{k_{\|} v_{\|}-\omega} \frac{e \phi}{T_{e}}
$$

In addition to correctly evaluate the resonant response of $\omega \approx k_{\|} v_{\|}$with this equation, we can also use it to calculate the nonadiabatic part associated with $k_{\|} v_{\|} \gg \omega$ accurately, whereas the corresponding expression obtained from the original weight equation, Eq. (4), i.e.,

$$
w \approx \frac{k_{\|} v_{\|}}{k_{\|} v_{\|}-\omega} \frac{e \phi}{T_{e}}
$$

has to satisfy $k_{\|} v_{\|} \Delta t<1$ in order to yield correctly the non-adiabatic response as well as the adiabatic response of Eq. (13). As stated earlier, one consequence of violating the Courant condition, Eq. (12), is that the plasma response will be inaccurate [1]. This is actually working to our advantage. In the present case, the inaccuracy results in the elimination of the $\omega_{H}$ mode in Eq. (7) by using large $\Delta t$ and, thereby, forcing the response of the fast electrons to be adiabatic, while still preserving the ion acoustic branch of Eq. (8). When smaller $\Delta t$ 's are used, $\omega_{H}$ modes can then be restored. Thus, physics alone dictates the choice of time steps used in the simulation. This is the most unique feature of the split-weight scheme.

To verify the validity of the split-weight scheme, let us study the case of one-dimensional drift waves similar to those given earlier [3]. From Eq. (1), the one-dimensional gyrokinetic Vlasov equation can be expressed as 


$$
\frac{d \delta f_{\alpha}}{d t} \equiv \frac{\partial \delta f_{\alpha}}{\partial t}+v_{\|} \frac{\partial \delta f_{\alpha}}{\partial x_{\|}}+s_{\alpha} v_{t \alpha}^{2}\left(\frac{\partial}{\partial x_{\|}} \frac{e \phi}{T_{e}}\right) \frac{\partial \delta f_{\alpha}}{\partial v_{\|}}=-\frac{c}{B} \kappa_{n} \frac{\partial \phi}{\partial y} F_{0 \alpha}+s_{\alpha} v_{\|}\left(\frac{\partial}{\partial x_{\|}} \frac{e \phi}{T_{e}}\right) F_{0 \alpha}
$$

where $y=\theta x_{\|}, \theta<<1$ is the angle between the external $B$ field and the $z$-axis, and $\boldsymbol{\kappa}_{\alpha}=\kappa_{n} \hat{\mathbf{x}}$ is the density inhomogeneity in the x-direction. The only difference between Eqs. (1) and (18) is the absence of the nonlinear $\mathbf{E} \times \mathbf{B}$ term for the one-dimensional case. Therefore, the basic equations for particle pushing now consist of Eq. (9) for the parallel particle motion, Eq. (3) for the particle acceleration, Eq. (4) for tracking the particle weights for the ions, Eq. (15) for the non-adiabatic part of the weights for the electrons, and Eqs. (16) and (17) for the field calculations. The dominant nonlinearity comes from the velocity space trapping in Eq. (3). In order to compare with Ref. [3], the simulation has been carried out on a 64-grid system by using 6765 particles and keeping only the first mode with $k_{y} \rho \approx 0.8$. The particle size is chosen to be zero and other parameters are: $m_{i} / m_{e}=1837, T_{e} / T_{i}=1$, $\kappa_{n} \rho_{s}=0.025, \theta=0.01$ and $\Omega_{i} \Delta t=0.2$. The simulation results shown in Fig. 1 are similar to those reported earlier [3] in terms of (a) the mode frequency $\left(\omega / \Omega_{i} \approx 0.09\right)$, (b) the growth rate $\left(\gamma / \Omega_{i} \approx 0.012\right)$ as well as the saturation amplitude $\left(\left|e \phi / T_{e}\right| \approx 0.9 \%\right)$, and (c) the nonlinear modification to the background Maxwellian, where $\delta h_{0 e}=\sum_{j=1, N} w_{j}^{N A} \delta\left(v_{\|}-v_{\| j}\right)$ is the consequence of mode coupling. The small time step used here with $\omega \Delta t \approx 0.018$ and $k_{\|} v_{t e} \Delta t \approx 0.07$ is to satisfy the conservation properties for the nonlinear simulation.

To demonstrate that the new scheme can indeed circumvent the Courant condition, let us carry out the linearized simulation with the same set of parameters by using Eqs. (9) and (10) for particle pushing, the linearized Eq. (4) with $v_{\|}=0$ for the ion weights, the linearized Eq. (15) with $v_{\|}=0$ for the electron weights, together with Eq. (16) and the linearized Eq. (17) for the fields. The results with 46368 particles using $\Omega_{i} \Delta t=8$ are shown in Fig. 2, where the real frequency remain unchanged but the growth rate goes up to $\gamma / \Omega_{i} \approx 0.016$. For this time step, we have $\omega \Delta t \approx 0.72$ and $k_{\|} v_{t e} \Delta t \approx 2.75$ as specified by Eq. (12). While simulations with larger number particles could reduce the discrepancy, using fewer particles give rise to unacceptably large growth rates. (Or, we can use a smaller time step, e.g., $\Omega_{i} \Delta t=5$ which gives $k_{\|} v_{t e} \Delta t \approx 1.72$ and $\gamma / \Omega_{i} \approx 0.012$ with 10946 particles.) The implication here is that, 
indeed we can violate the Courant condition, but we have to use larger number of particles to minimize the noise caused by the fast particles. However, this should not be a hindrance for simulation in three-dimensional toroidal geometry, where millions of particles are used and the fluctuations are dominated by long wavelength modes $[7,11]$.

In conclusion, the proposed split-weight scheme seems to work well for low frequency microinstabilities and its extension to include finite- $\beta$ and toroidal effects will be reported elsewhere. We believe this work represent an important step toward realistic 3D simulations of microturbulence and gyrokinetic-MHD physics in tokamaks and stellarators. The methodology can be applied to space physics and unmagnetized plasmas as well.

The authors would like to acknowledge Dr. Jerome Lewandowski for his careful reading of the manuscript. One of us (I.M.) would also like thank Dr. Harry Mynick and Dr. Scott Parker for their support and discussions. This work is supported by the U.S. Department of Energy. 


\section{REFERENCES}

[1] A. B. Langdon, J. Comput. Phys. 30, 202 (1979).

[2] A. M. Dimits, and W. W. Lee, J. Comput. Phys. 107, 309 (1993).

[3] S. E. Parker, and W. W. Lee, Phys. Fluids B 5, 77 (1993).

[4] W. W. Lee Lee, Phys. Fluids 26, 556 (1983).

[5] W. W. Lee, J. Comput. Phys. 72, 243 (1987).

[6] W. W. Lee and W. M. Tang, Phys. Fluids 31, 612 (1988).

[7] Z. Lin, T. S. Hahm, W. W. Lee, W. M. Tang, and R. White, Science 281, 1835 (1998).

[8] C. W. Nielson and H. R. Lewis, Methods of Computational Physics, Vol. 16 (Academic Press), 976 (1976).

[9] E. A. Frieman and L. Chen, Phys. Fluids 25, 502 (1982).

[10] W. W. Lee, J. A. Krommes, C. Oberman, and R. A. Smith, Phys. Fluids 27, 2652 (1984).

[11] W. W. Lee and R. A. Santoro, Phys. Plasmas 4, 169 (1997). 


\section{FIGURES}
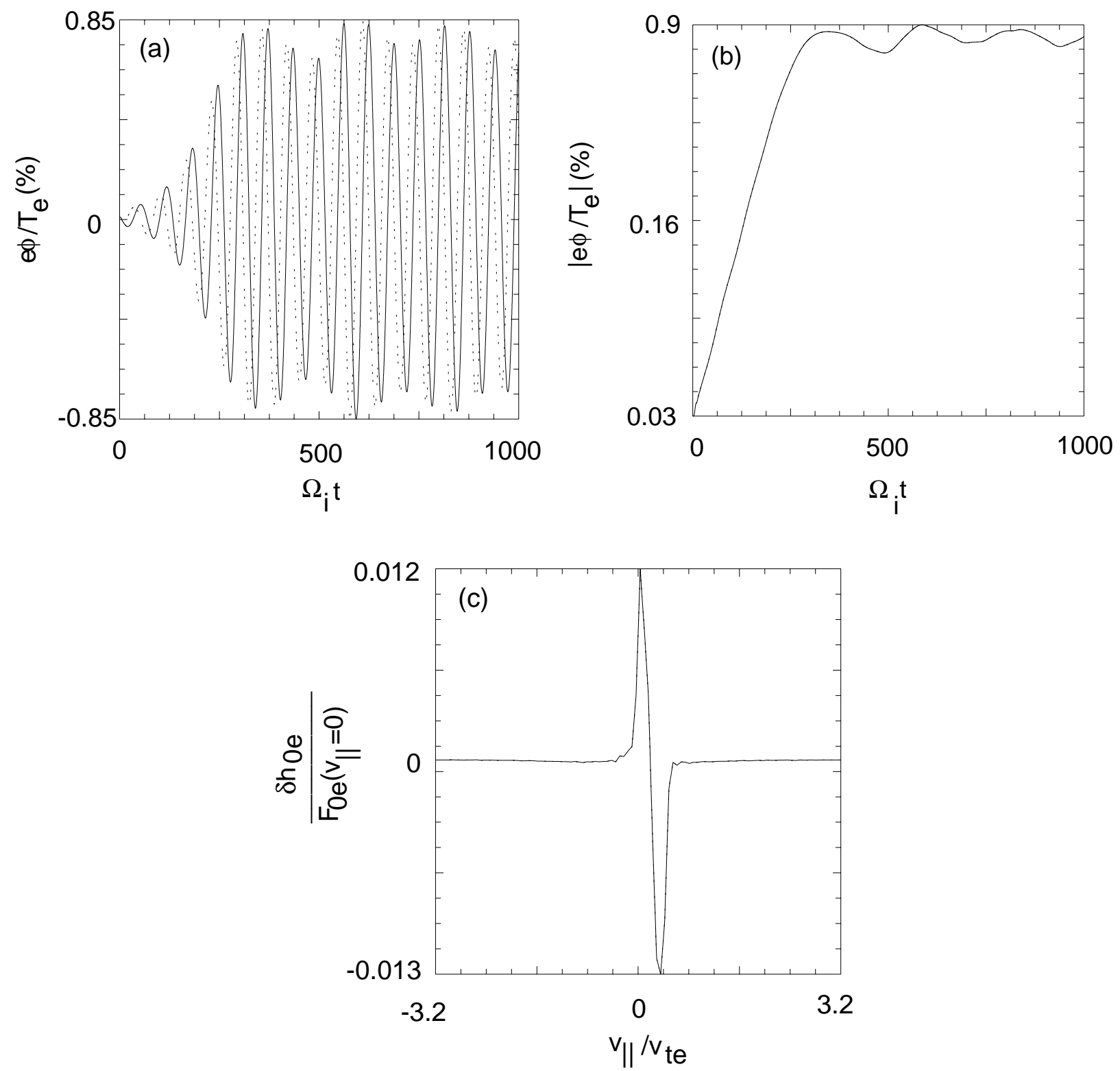

FIG. 1. Nonlinear simulation with 6765 particles for $k_{\perp} \rho_{s} \approx 0.8, k_{\|} / k_{\perp}=0.01$ on a 64 -grid system. (a) The time history for the real (solid line) and imaginary (dashed line) parts of the electrostatic potential, (b) the corresponding amplitude evolution, and (c) the second order perturbed electron distribution. 

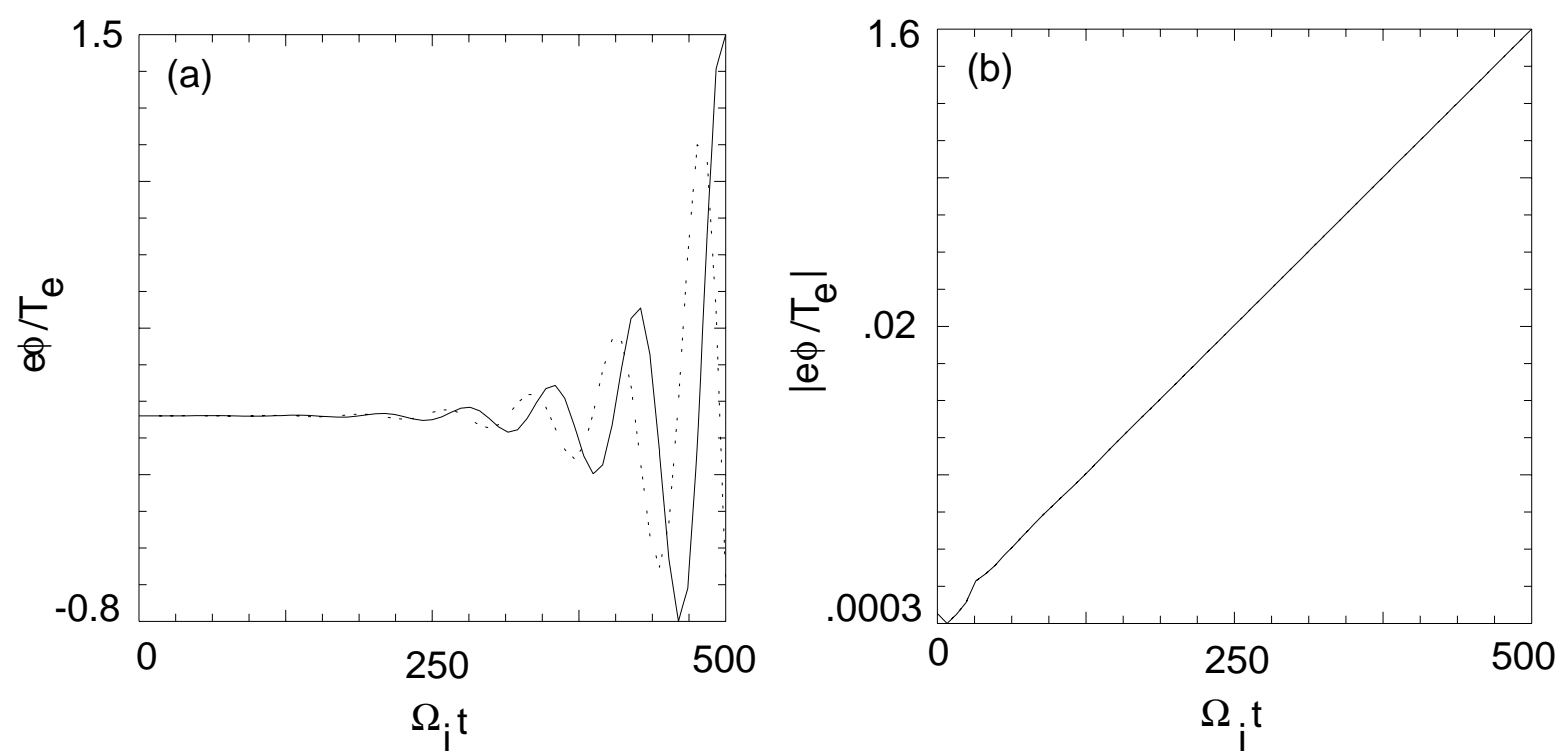

FIG. 2. Linear simulation with 46386 particles for $k_{\perp} \rho_{s} \approx 0.8, k_{\|} / k_{\perp}=0.01$ on a 64 -grid system for $k_{\|} v_{t e} \Delta t>1$. (a) The time history for the real (solid line) and imaginary (dashed line) parts of the electrostatic potential, and (b) the corresponding amplitude evolution. 